\title{
Erratum to: A donor thrombomodulin gene variation predicts graft-versus-host disease development and mortality after bone marrow transplantation
}

\author{
Haruka Nomoto ${ }^{1}$ Akiyoshi Takami ${ }^{2} \cdot$ J. Luis Espinoza $^{3} \cdot$ Keitaro Matsuo $^{4} \cdot$ \\ Shohei Mizuno $^{2}$ - Makoto Onizuka ${ }^{5} \cdot$ Koichi Kashiwase $^{6} \cdot$ Yasuo Morishima $^{7}$. \\ Takahiro Fukuda $^{8}$ - Yoshihisa Kodera ${ }^{9}$ Noriko Doki ${ }^{10}$ - Koichi Miyamura ${ }^{11}$.

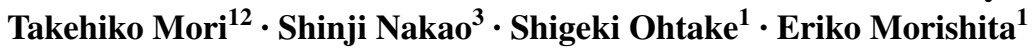

Published online: 9 September 2015

(C) The Japanese Society of Hematology 2015

\section{Erratum to: Int J Hematol}

\section{DOI 10.1007/s12185-015-1852-7}

Unfortunately, Dr. Shohei Mizuno's affiliation has been incorrectly published in the original publication of the article. The correct affiliation should read as "Division of Hematology, Department of Internal Medicine, Aichi Medical University School of Medicine, 1-1 Yazakokarimata, Nagakute 480-1195, Japan".

The online version of the original article can be found under doi:10.1007/s12185-015-1852-7.

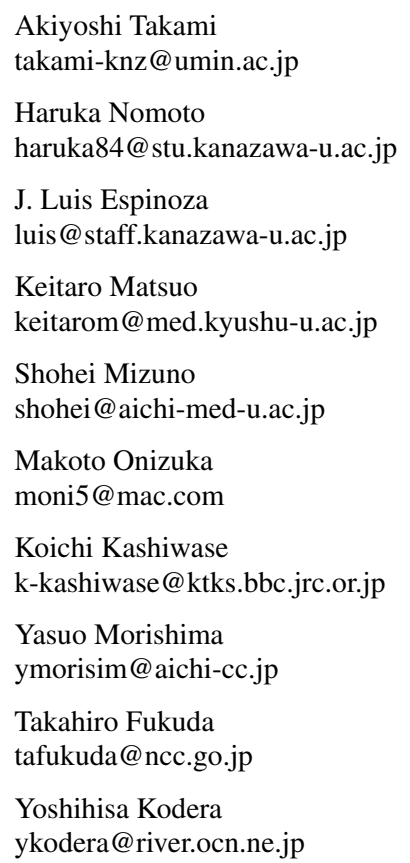

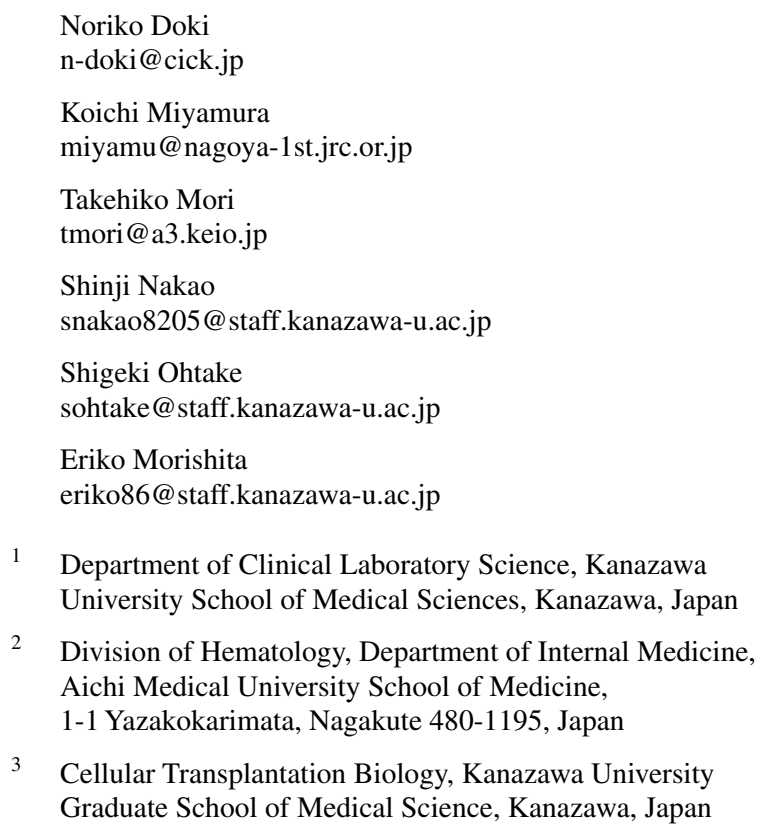


4 Department of Preventive Medicine, Kyushu University Faculty of Medical Sciences, Fukuoka, Japan

5 Department of Hematology and Oncology, Tokai University School of Medicine, Isehara, Japan

6 Japanese Red Cross Kanto-Koshinetsu Block Blood Center, Tokyo, Japan

7 Division of Epidemiology and Prevention, Aichi Cancer Center Research Institute, Nagoya, Japan

8 Hematopoietic Stem Cell Transplantation Unit, National Cancer Center Hospital, Tokyo, Japan

9 Department of Promotion for Blood and Marrow Transplantation, Aichi Medical University, Nagoya, Japan
10 Hematology Division, Tokyo Metropolitan Cancer and Infectious Diseases Center Komagome Hospital, Tokyo, Japan

11 Department of Hematology, Japanese Red Cross Nagoya First Hospital, Nagoya, Japan

12 Division of Hematology, Department of Medicine, Keio University School of Medicine, Tokyo, Japan 